\title{
CRECIMIENTO DE GORRINOS PROVENIENTES DE MADRES CON Y SIN ANTECEDENTES DE VACUNACIÓN CONTRA Mycoplasma hyopneumoniae
}

\author{
Jannet Cisneros S. ${ }^{1}$, Sonia Calle E. ${ }^{2}$, Marlon Torres A. ${ }^{3}$, Néstor Falcón P. ${ }^{4}$, \\ Miguel Morales C. ${ }^{2}$ y Francisco Acosta Ch. ${ }^{1}$
}

\section{Abstract}

The aim of the study was to evaluate body weight gain of piglets coming from vaccinated and not vaccinated sows against Mycoplasma hyopneumoniae. Two groups of four sows each from a herd positive to $M$. hyopneumoniae were randomly selected. One group was vaccinated with a bacterin against M. hyopneumoniae at day 85 of gestation, while the other was kept as control. After parturition, 30 piglets ( $50 \%$ female and $50 \%$ male) from each group of sows were selected and weighed at weaning (19 days of age) and at slaughter (149 days of age). Food intake per group was daily recorded. In addition, the degree of lung lesions at slaughter in a scale of 0-6 was evaluated. No significant statistical difference was found in body growth (final body weight and body weight gain) as well as in lung lesions between groups or sex.

Key words: Mycoplasma hyopneumoniae, pig, productive parameters, lung lesions

\section{Resumen}

El presente estudio tuvo por objetivo evaluar la ganancia de peso de un lote de gorrinos provenientes de madres vacunadas y no vacunadas contra Mycoplasma hyopneumoniae. Se seleccionaron al azar 2 grupos de cuatro marranas multíparas provenientes de una granja positiva a $M$. hyopneumoniae. Un grupo fue vacunado con una bacterina contra $M$. hyopneumoniae en el día 85 de la gestación y el otro se dejó sin vacunar quedando como control. Luego del parto, se seleccionaron 30 lechones (50\% hembras y 50\% machos) de cada grupo de madres. Los animales se pesaron al destete (19 días de edad) y al sacrificio (149 días). El consumo de alimento por grupo de tratamiento fue registrado diariamente. Además, se evaluó el grado de lesión pulmonar en los animales beneficiados en una escala arbitraria del 0 al 6 . No se encontró diferencia estadística significativa en el crecimiento (peso final y ganancia de peso) ni en lesiones pulmonares entre grupos o sexo.

Palabras clave: Mycoplasma hyopneumoniae, cerdo, parámetros productivos, lesiones pulmonares

\footnotetext{
${ }^{1}$ Laboratorio de Vida y Fauna Silvestre, FMV-UNMSM

${ }^{2}$ Laboratorio de Microbiología y Parasitología Veterinaria, FMV-UNMSM

${ }^{3}$ Laboratorio de Zootecnia y Producción Agropecuaria, FMV-UNMSM

${ }^{4}$ Laboratorio de Medicina Veterinaria Preventiva, FMV-UNMSM
} 


\section{INTRODUCCIÓN}

Las neumonías son una de las principales enfermedades causantes de pérdidas económicas en las granjas porcinas tecnificadas. Dentro de los agentes causantes de neumonías, el Mycoplasma hyopneumoniae se ha convertido en el patógeno más importante del Complejo Respiratorio Porcino junto con el virus del Síndrome Reproductivo y Respiratorio Porcino (SRRP) (Halbur, 1997). Este patógeno tiene la capacidad de actuar sinérgicamente con otros agentes infecciosos (Ibarra, 2000) como Pasteurella multocida, Streptococcus suis, Actinobacillus pleuropneumoniae y posiblemente también con el Circovirus tipo 2 en la Neumonía Intersticial (Ohlinger et al., 2002).

La importancia económica de esta enfermedad radica en que afecta el crecimiento de los gorrinos y afecta la conversión alimenticia ocasionando lotes desiguales y retraso para alcanzar el peso de mercado (Camacho y Calle, 2003). Straw (1989) determinó que la ganancia de peso disminuye cerca de 25 gramos diarios por cada $10 \%$ de tejido pulmonar afectado por neumonía, siendo las pérdidas de peso más importantes en cerdos afectados a una edad temprana (Morris et al., 1995), aunque Thacker y Thacker (1999) afirman que la infección es más severa en lechones mayores de 8 semanas que en menores de 6 semanas de edad.

En el Perú se ha reportado $12 \%$ de reactores a Mycoplasma hyopneumoniae en cerdos de crianza artesanal (Ibarra, 2000) y de $25 \%$ en animales antes del beneficio (Huallanca, 1999).

La vacunación contra $M$. hyopneumoniae ha permitido reducir los efectos perjudiciales de esta enfermedad (Thacker y Thacker, 2000). Jayappa et al. (2001) y Ruiz y Pijoan (2002) demostraron que la vacunación en madres gestantes permite una mayor transmisión de anticuerpos maternales a los lechones, previniendo el desarrollo de lesio- nes producidas por $M$. hyopneumoniae hasta las 6 semanas de edad.

La transmisión pasiva de anticuerpos maternos a las crías varía de acuerdo al estado inmunológico de la madre. Se ha demostrado que los anticuerpos transmitidos por madres no vacunadas y expuestas naturalmente a $M$. hyopneumoniae descienden a la sexta semana de edad, mientras que los transmitidos por las madres vacunadas persisten hasta la octava semana (Calle et al., 2003). El objetivo del presente trabajo fue determinar si la vacunación contra $M$. hyopneumoniae en marranas influye en el comportamiento productivo de sus crías.

\section{Materiales y Métodos}

\section{Lugar de estudio}

El estudio se realizó en una granja porcina tecnificada localizada en el distrito de Puente Piedra, Lima, entre los meses de mayo y noviembre del 2003. Las inspecciones de los pulmones se realizaron en el camal José Olaya, Lurín.

La granja es de producción en un solo sitio, con el sistema "todo dentro-todo fuera". Tiene un plantel reproductor de 600 madres que son vacunadas al día 85 de gestación contra Escherichia coli y Clostridium sp. En un estudio previo se determinó que el $58 \%$ de los animales de la granja tenía anticuerpos contra Mycoplasma hyopneumoniae.

\section{Manejo de los animales experimentales}

Se utilizaron 2 grupos de 4 marranas escogidas al azar. Un grupo fue vacunado con una bacterina contra Mycoplasma hyopneumoniae a los 85 días de gestación, mientras que el otro sirvió como grupo control. Las marranas eran multíparas de 2 y 3 partos, en aparente buen estado de salud y sin antecedentes de enfermedad al inicio del 
experimento. Al parto, el promedio de lechones por camada fue de 10 animales, de los cuales se seleccionaron 30 por grupo (mitad hembras y mitad machos) para el seguimiento de anticuerpos maternales y desarrollo corporal. El tamaño muestral de lechones se calculó con la fórmula de la diferencia de medias, resultando 12 animales por cada tratamiento.

Se verificó que los lechones hayan ingerido el calostro de su madre. El periodo de lactación fue de $19 \pm 2$ días. En el área de recría permanecieron hasta los 70 días de edad y en el área de engorde se quedaron hasta que obtuvieron el peso de beneficio $(90 \mathrm{~kg}$ de peso vivo), que en promedio fue a los 145 días de edad. Se siguió el manejo sanitario y de alimentación de la granja.

\section{Recolección y análisis de datos}

Los animales se pesaron el día del destete y el día que fueron llevados al camal. El consumo de alimento se registró diariamente y por grupo de tratamiento. Con los datos de consumo de alimento promedio y la ganancia de peso promedio entre el destete y el beneficio se calculó el índice de conversión media.

Se evaluó el grado de lesión pulmonar en los animales beneficiados. Se examinaron los 7 lóbulos de cada pulmón, asignándoles una puntuación a cada lóbulo de acuerdo al porcentaje de consolidación pulmonar (Piffer y Britto, 1991). Para esto, se mide la extensión de la lesión de consolidación pulmonar en cada lóbulo (expresada en \%) y se calcula el área pulmonar consolidada por lóbulo, tomando en cuenta su peso relativo, y se suma cada parte para obtener el área pulmonar consolidada total. El área de consolidación se agrupó en 6 categorías, siguiendo el modelo propuesto por Piffer y Britto (1991).

\section{Análisis estadístico}

Para la evaluación de los pesos finales y la ganancia de peso promedio de los gorrinos provenientes de madres vacunadas y los de madres no vacunadas se utilizó la prueba de " $t$ " Student de independencia.

Se utilizó la prueba de Mann-Whitney para determinar el efecto de la condición inmunológica de la madre sobre la consolidación pulmonar en los gorrinos.

\section{Resultados}

El peso inicial de los gorrinos hembras provenientes de madres no vacunadas fue estadísticamente superior al de los gorrinos provenientes de madres vacunadas $(\mathrm{p}<0.05)$, en tanto que los pesos de los machos fueron estadísticamente similares.

Se tuvo que descartar un animal del grupo control por un problema sanitario ajeno al estudio, por lo que se retiró a otro animal similar del grupo vacunado para balancear los grupos.

No se encontró diferencia estadística significativa en el peso a la edad de beneficio ni en la ganancia de peso entre el destete y el beneficio entre gorrinos provenientes de madres vacunadas y no vacunadas contra $M$. hyopneumoniae. Tampoco se encontraron diferencias debidas a sexo (Cuadro 1); sin embargo, se encontró una mayor homogeneidad en los pesos de los animales provenientes de madres vacunadas.

El consumo de alimento promedio durante el periodo experimental fue de $197.8 \mathrm{y}$ $212.2 \mathrm{~kg}$ por gorrino provenientes de madres vacunadas y no vacunadas contra $M$. hyopneumoniae, respectivamente, de allí que la conversión alimenticia para estos grupos fue de 2.3 y 2.5 , respectivamente.

No presentaron lesiones pulmonares el 20.7 y $24.1 \%$ de los gorrinos provenientes de madres vacunadas y no vacunadas contra $M$. hyopneumoniae. No se encontró diferencia estadística significativa al evaluar el grado de 
Cuadro 1. Peso corporal al destete y al beneficio $(\mathrm{kg})$ y ganancia de peso en ese periodo $(\mathrm{kg})$ en gorrinos provenientes de marranas vacunadas y no vacunadas contra $M$. hyopneumoniae

\begin{tabular}{cccc}
\hline & $\mathrm{N}^{\mathrm{o}}$ & $\begin{array}{c}\text { Gorrinos de Madres } \\
\text { Vacunadas }\end{array}$ & $\begin{array}{c}\text { Gorrinos de Madres No } \\
\text { Vacunadas }\end{array}$ \\
\hline Peso inicial (destete) & 60 & $5.9 \pm 0.2$ & $6.9 \pm 0.4$ \\
- Machos & 30 & $6.2 \pm 0.6$ & $6.8 \pm 1.1$ \\
- Hembras & 30 & $5.9 \pm 0.6$ & $7.2 \pm 1.0$ \\
Peso final (edad de beneficio) & 58 & $92.4 \pm 8.1$ & $91.6+9.7$ \\
- Machos & 29 & $96.5 \pm 7.9$ & $93.1 \pm 10.2$ \\
- Hembras & 29 & $88.0 \pm 5.8$ & $90.3 \pm 9.3$ \\
Ganancia de peso & 58 & $86.5 \pm 8.2$ & $86.7 \pm 10.1$ \\
- Machos & 29 & $90.3 \pm 8.1$ & $83.1 \pm 9.9$ \\
- Hembras & 29 & $82.1 \pm 6.1$ & \\
\hline
\end{tabular}

Cuadro 2. Grado de consolidación pulmonar $^{1}$ en gorrinos beneficiados provenientes de madres vacunadas y no vacunadas contra $M$. hyopneumoniae

\begin{tabular}{|c|c|c|c|c|}
\hline \multirow{3}{*}{ Grado $^{2}$} & \multicolumn{4}{|c|}{ Gorrinos Provenientes de Madres } \\
\hline & \multicolumn{2}{|c|}{ No Vacunadas } & \multicolumn{2}{|c|}{ Vacunadas } \\
\hline & $\mathrm{N}^{\mathrm{o}}$ & $\%$ & $\mathrm{~N}^{\mathrm{o}}$ & $\%$ \\
\hline 0 & 7 & 24.1 & 6 & 20.7 \\
\hline 1 & 15 & 51.7 & 16 & 55.2 \\
\hline 2 & 4 & 13.8 & 7 & 24.1 \\
\hline 3 & 2 & 6.9 & 0 & 0 \\
\hline 4 & 0 & 0 & 0 & 0 \\
\hline 5 & 1 & 3.5 & 0 & 0 \\
\hline 6 & 0 & 0 & 0 & 0 \\
\hline Total & 29 & 100.0 & 29 & 100.0 \\
\hline
\end{tabular}


consolidación pulmonar en los cerdos; sin embargo, hubo algunos gorrinos de madres no vacunadas que presentaron grados de lesión 3 y 5, situación que no se presentó en gorrinos de madres vacunadas (Cuadro 2). Tampoco se observaron diferencias significativas por efecto del sexo del animal en el grado de consolidación pulmonar.

\section{Discusión}

Los promedios de pesos a la edad del beneficio y la ganancia promedio entre el destete y el final del periodo experimental fueron similares en los gorrinos provenientes de madres vacunadas y no vacunadas contra M. hyopneumoniae.

Los animales del presente estudio se sometieron a un reto de campo donde los anticuerpos contra $M$. hyopneumoniae que fueron transferidos por las madres provienen de infecciones que pudieron haber sido adquiridas en diferentes momentos e incluso pudo haberse dado el caso que algunas madres no se hayan llegado a infectar. Esto podría explicar la nula diferencia en desarrollo corporal de los gorrinos entre ambos grupos experimentales, diferencias que sí han sido demostradas por otros autores (Clark et al., 1993; Rautiainen et al., 2000; Sitjar et al., 1996). No obstante, hay reportes de otras investigaciones que tampoco pudieron demostrar el efecto benéfico en los gorrinos de la vacunación de las madres (Thacker y Thacker, 2001; Escobar et al., 2002).

Los gorrinos provenientes de madres vacunadas contra $M$. hyopneumoniae tuvieron una mejor conversión alimenticia (2.3) que aquellos de madres no vacunadas (2.5), lo que significó un ahorro cercano a los $15 \mathrm{~kg}$ de alimento por animal. Al respecto, Maes et al. (2000) demostraron que los animales protegidos contra la enfermedad tienen una mejor conversión alimenticia, aunque Escobar et al. (2002) no encontraron cambios en el consumo de alimento en animales infectados experimentalmente con $M$. hyopneumoniae, ni siquiera cuando comenzaron a mostrar síntomas clínicos. Al parecer, las citoquinas que se liberan por la infección con $M$. hyopneumoniae no son suficientes para disminuir el apetito o afectar el crecimiento de los animales.

La literatura reporta que cerdos de 4 a 6 semanas de edad, nacidos de madres vacunadas, tienen mayor protección contra la consolidación pulmonar luego del reto experimental (Wasmoen et al., 2000). Asimismo, se ha determinado que la prevalencia de lesiones neumónicas encontradas en cerdos beneficiados eran mayores si los animales convertían tardíamente (Rautiainen et al., 2000). No obstante, en el presente estudio no se llegó a encontrar diferencias significativas entre ambos grupos experimentales.

\section{Conclusiones}

No se encontró diferencias significativas en la ganancia de peso entre el destete y la edad de beneficio ni en el grado de lesión pulmonar al beneficio en gorrinos provenientes de madres vacunadas y no vacunadas contra $M$. hyopneumoniae.

\section{Literatura Citada}

1. Calle, S.; C. Camacho; M. Torres. 2003. Inmunidad natural e inducida contra Mycoplasma hyopneumoniae medida desde el nacimiento hasta la edad de mercado en cerdos bajo crianza intensiva. Mundo Avícola y Porcino 44: 48-49.

2. Camacho, C.; S. Calle. 2003. Neumonía enzoótica porcina. Mundo Avícola y Porcino 45: 48-50.

3. Clark, L.; C. Armstrong; A. Scheidt; W.G. VanAlstine. 1993. The effect of Mycoplasma hyopneumoniae infection on growth in pigs with or without environmental constraints. J. Swine Health Prod. 1: 10-14. 
4. Escobar, J.; W. Van Alstine; D. Baker; $\boldsymbol{R}$. Jhonson. 2002. Growth performance and whole-body composition of pigs experimentally infected with Mycoplasma hyopneumoniae. Am. Soc. Anim. Sci. 80: 384-391.

5. Halbur, P. 1997. Making the diagnosis with serology, antigen detection and PCR. Proc. Iowa State University Veterinary Medicine Seminar. p 3-7. Iowa, USA.

6. Huallanca, A. 1999. Determinación de reactores positivos a Mycoplasma hyopneumoniae en cerdos sacrificados en un camal frigorífico. Tesis de Médico Veterinario. Facultad de Medicina Veterinaria, Univ. Nacional Mayor de San Marcos. Lima. 53 p.

7. Ibarra, M. 2000. Evidencia de la presencia de Mycoplasma hyopneumoniae en cerdos provenientes de granja de crianza artesanal. Tesis de Médico Veterinario. Facultad de Medicina Veterinaria, Univ. Nacional Mayor de San Marcos. Lima. 60 p.

8. Jayappa, H.; R. David; V. RappGabrielson; T. Wasmoen; E. Thacker; B. Thacker. 2001. Evaluation of the efficacy of Mycoplasma hyopneumoniae bacteria following immunization of young pigs in the presence of varying levels of maternal antibodies. Proc. Annual Meeting Am. Assoc. Swine Vet. p 237-241. Nashville, USA.

9. Maes, D.; M. Verdonek; W. Verbeke; J. Viaene; A. de Kruij. 2000. Mycoplasma hyopneumoniae: Benefit to cost of vaccinations. Am. Assoc. Swine Practitioners 32: 327- 333.

10. Morris, C.; I. Gardner; S. Heitala; T. Carpenter; R. Anderson; K. Parker. 1995. Seroepidemiologic study of natural transmission of Mycoplasma hyopneumoniae in a swine herd. Prev. Vet. Med. 21: 323-333.
11. Ohlinger, V.; K. Pesch; C. Seller. 2002. Four diseases linked to porcine circovirus II. Pig Internat. 32: 17-20.

12. Piffer, I.; J. Brito. 1991. Descricao de um modelo para valiacao e quantificacao de lesoes pulmonares de suinos e formulacao de um indice para classificacao dos rebanhos. Concordia, SC. EMBRAPA-CPNSA. 12 p.

13. Rautiainen, E.; A. Virtala; $P$. Wallgren; H. Saloniemi. 2000. Varying effects of infections with Mycoplasma hyopneumoniae on the weight gain recorded in three different multi-source fattening pig herds. J. Vet. Med. B. Infect. Dis. Vet. Public Health 47: 461-469.

14. Ruiz, A.; C. Pijoan. 2002. Efectos de la vacunación sobre la prevalencia de Mycoplasma hyopneumoniae. Memorias I Congreso Latinoamericano de Suinocultura. p 5-6. Foz de Iguazú.

15. Sitjar, M.; E. Noyes; X. Simon; C. Pijoan. 1996. Relationship among seroconversion to Mycoplasma hyopneumoniae, lung lesions and production parameters in pigs. Swine Health Prod. 4: 273-277.

16. Straw, B. 1989. Estimation of the cost of pneumonia in swine herds. J. Am. Vet. Med. Assoc. 195: 1702-1706.

17. Thacker, B.; E. Thacker. 2001. Influence of maternally-derived antibodies on the efficacy of a Mycoplasma hyopneumoniae bacterin. Proc. $32^{\text {nd }}$ Annual Meeting Am. Assoc. Swine Vet. p 513-515. Nashville, USA.

18. Wasmoen, T.; V. Rapp-Gabrielson; $R$. David; R. Jayappa. 2000. Evaluation of maternal antibody interference against active immunization of young pigs form Mycoplasma hyopneumoniae. Proc. $81^{\text {st }}$ Conf. Research Workers in Animal Disease. Chicago. Abstract: 111. 